\title{
Semi-analytical model of the vertical impact of a 316 stainless steel rod
}

\author{
Xuan Ye ${ }^{\mathrm{a}, \mathrm{b}}$, Jianqiao $\mathrm{Hu}^{\mathrm{a}, \mathrm{b}}$, Jingru Song ${ }^{\mathrm{a}, \mathrm{b}}$, Xiaoming Liu ${ }^{\mathrm{a}, \mathrm{b}, *}$, Yueguang $\mathrm{Wei}^{\mathrm{c}}$ \\ ${ }^{a}$ LNM, Institute of Mechanics, Chinese Academy of Sciences, Beijing 100190, China \\ ${ }^{\mathrm{b}}$ School of Engineering Science, UCAS, Beijing 100049, China

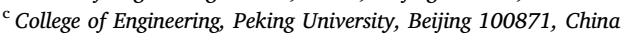

\section{A R T I C L E I N F O}

\section{Keywords:}

Vertical impact

Length/diameter ratio

Elasto-plastic contact

Equivalent spring model

\begin{abstract}
A B S T R A C T
A semianalytical model was proposed to study the vertical impact of a slender rod striking a rigid flat surface. Within the semianalytical model, we considered the longitudinal vibration of the rod by using continuum mechanics while employing a plastic contact model, an equivalent spring model, to describe the contact of the linear hardening hemispherical tip and a rigid flat surface. The predicted displacement and velocity histories along the rod agreed well with the corresponding finite element simulations. In addition, from the proposed semianalytical model, we determined that there was a critical length/diameter ratio that can be used for any plastic contact of a linear hardening rod. The critical length/diameter ratio was approximately 17 for a rod made of 316 stainless steel. Below this critical length/diameter ratio, the rod behaved as a rigid body, i.e., the velocities of different sections of the rod reversed simultaneously during the impact. Above this critical length/ diameter ratio, the velocity of the top section reversed much earlier than the other sections of the rod; hence, the slenderness effect should be accounted for in these conditions.
\end{abstract}

\section{Introduction}

Understanding rod contact and impact is important in structural design [1-4]. For instance, as discussed in the literature [5], rod contact will cause fretting failure when heat exchanger tubes contact loose baffle plates. Another typical rod impact case is the drop-induced failure of nuclear fuel rods during transportation and lifting [6]. The contact or impact loads on the rod produced by these transient collisions can create significant risks for the safe operation of the system. Generally, these impacts occur over a short time interval of microseconds to milliseconds, and complex dynamic responses occur during these short periods. Because these contacts are often accompanied by geometric, material, and contact nonlinearities, the rod responses which have a significant effect on structural safety, are very hard to predict.

Various experimental [7-12], numerical [13,14], and analytical $[10,15,16]$ studies have been employed to assess the impact of rods with flat surfaces. Due to the cost of experiments, many studies have focused on determining theoretical solutions to impact problems. Although the finite element method (FEM) can be used to simulate these dynamic impact events, it is only able to deal with specific situations, and there are also additional problems with non-convergence of the solutions using numerical simulations. Other studies, as discussed in references $[9,10]$, have coupled the rigid body motion of rods with the contact forces produced at the interfaces of the rod tips and rigid substrates and solved Newton's equations to describe the motion of the rods.

The contact between a hemisphere and a flat surface has been investigated through the use of various classical continuum models. For fully elastic impacts, the contact is assumed to follow the Hertz theory of elastic contact [17]. For elastic-plastic contacts, different models have been employed depending on the particular problem. In the Kogut-Komvopoulos (KK) [18], Ye-Komvopoulos (YK) [19], Brake [20], Stronge [21], Thornton [22], and modified Jackson-Green (MJG) [9] models, a rigid hemisphere in contact with a deformable half-space was considered. In the Jackson-Green (JG) [23] and Kogut-Etsion (KE) [24] models, the contact of an elastic-plastic hemisphere with a rigid flat surface was considered, but the latter formula is simpler. The KE contact model divides the contact into two subphases during loading: the fully elastic phase when $\delta / \delta_{\mathrm{c}} \leq 1$, where $\delta$ is the interference and $\delta_{\mathrm{c}}$ is the interference at which the yields start, and the elasto-plastic phase when $1<\delta / \delta_{\mathrm{c}} \leq 110$. For these models, the deformable material was assumed to be elastic-perfectly plastic. In references [25,26], strain hardening effects were employed, Hertz contact theory was used for the elastic phase, and Meyer's hardness was used for the plastic phase. However, for some impact problems, such as long rod impacts, $\delta / \delta_{\mathrm{c}}$ may be greater than 110, and strain hardening plasticity cannot be ignored; thus, further efforts are required to establish new impact

\footnotetext{
* Corresponding author.

E-mail address: xiaomingliu@imech.ac.cn (X. Liu).
} 


\author{
List of symbols \\ $v_{0}, v, v_{\text {Top }}$ Initial striking velocity, velocity, and velocity at the top \\ point \\ $v_{0, K E} \quad$ Maximum initial striking velocity applicable for the $\mathrm{KE}$ \\ model \\ $\Delta v \quad$ Velocity difference between the top and bottom points \\ $l \quad$ Length of the straight section of the rod \\ $l_{c} \quad$ Critical length of the straight section of the rod (for $l>l_{c}$, \\ the vibration effect must be considered) \\ $R, d=2 R$ Radius and diameter of the rod and hemispherical tip \\ $L=l+d \quad$ Total rod length \\ $s \quad$ Coordinate system along the rod axis \\ F $\quad$ Contact force \\ $\delta \quad$ Interference \\ $\delta_{\mathrm{c}} \quad$ Critical elastic interference \\ $\delta_{\mathrm{m}} \quad$ Maximum nonlinear interference \\ $\delta_{\mathrm{u}} \quad$ Ultimate interference \\ $k \quad$ Equivalent stiffness
}

\section{$K \quad$ Equivalent modulus}

$u \quad$ Longitudinal motion of the rod

$t \quad$ Time

$t_{\text {Bottom }} t_{\text {Top }}$ Difference in velocity reversal time between the bottom and top points

$c \quad$ One-dimensional longitudinal wave velocity

E Young's modulus

$A=\pi R^{2} \quad$ Cross-sectional area

$\omega, \omega_{n} \quad$ Natural frequency and $n$-order natural frequency

$\beta=\frac{\omega}{c} l, \beta_{n}=\frac{\omega_{n}}{c} l$ Parameters related to natural frequency and norder natural frequency

$a_{1}, a_{2}, b_{1}, b_{2}$ Constants that depend on the boundary and initial conditions

$a_{1 n}, b_{1 n}, b_{2 n}, c_{n}=a_{1 n} b_{2 n} \quad$ n-order constants that depend on the boundary and initial conditions

$m \quad$ Mass of the rod

$\rho \quad$ Material density of the rod

$v \quad$ Poisson's ratio of the rod models.

Other discrete models are also used to study the dynamic impact of a rod. In reference [27], a rod was discretized using a rigid body system connected by either spiral springs or a pair of linear springs. In addition, the impact was assumed to be frictionless, and the structural response was modeled by solving Newton's law. In references [28,29], a discrete model of a rod was proposed to obtain the equations of motion during impact by discretizing the rod into $n$ segments connected through viscoelastic links between neighboring pairs of segments. The internal vibrations of the rod and multiple impacts were observed to be primary factors associated with variations in the motion parameters.

The majority of the theoretical models discussed here are only applicable to relatively short and thick rods. For instance, in references $[9,10]$, the rod had a length of $304 \mathrm{~mm}$ with a diameter of $17.6 \mathrm{~mm}$. However, the aspect ratio (i.e., length/diameter ratio) of a long rod under various conditions cannot be ignored, and a typical rod used for a nuclear assembly has an aspect ratio of approximately 40 , as discussed in references [30,31], and the vibration response during impact cannot be ignored. In the present study, a theoretical model was developed to assess the vertical impact of 316 stainless steel (SS316) rods with a large aspect ratio. The proposed semianalytical model considered both the plastic contact of the rod tip and the vibration effect within the rod. Moreover, motion parameters such as displacement, velocity, and velocity reversal time were considered in the present study.

\section{Dynamics of vertical impact of rods}

\subsection{Impact configuration}

Fig. 1 shows a schematic of a rod vertically dropping onto a flat rigid surface with an initial striking velocity of $v_{0}$. The rod has a total length of $L=l+d$ and consists of three parts, a straight section with a length of $l$, and two hemispherical ends, each with a radius of $R$ such that $d=2 R$. In Fig. 1, a coordinate system $s$ is defined along the rod axis, where $s=0$ is the top end of the straight section of the rod neglecting the hemispherical top, $s=l / 2$ is the center point, and $s=l$ is the bottom of the straight section of the rod neglecting the hemispherical bottom. The rod was assumed to be SS316, for which the true tensile stressstrain relationship is given in Fig. 2. This true stress versus strain curve was obtained using a commercial mechanical testing system (MTS) and illustrates that SS316 has an elastic modulus of $162.67 \mathrm{GPa}$, a yield strength of $247.26 \mathrm{MPa}$, and a plastic linear hardening modulus of 1.93 GPa.

\subsection{Long rod impact model}

In Section 2.2.1, the dynamic vertical impact of a rod with an initial striking velocity of $4 \mathrm{~m} / \mathrm{s}$ was simulated using ABAQUS Explicit FEM to model a drop height of $0.8 \mathrm{~m}$. In Section 2.2.2, the static flattening of hemispheres with a flat rigid surface was simulated using ABAQUS Standard, for which the details are given by Fig. A.1 in Appendix A, and the interference was obtained from the dynamic impact simulations in Section 2.2.1. Based on the fact that both the dynamic impact and the static flattening simulations exhibited a linear relationship between the contact force and interference, an equivalent linear spring model was proposed to describe the contact of the hemispherical end with the rigid flat surface. From this result, a theoretical model for the vertical impact of a slender rod with hemispherical ends was developed in Section 2.2.3.

\subsubsection{Dynamic vertical impact of rods}

The dynamic vertical impact of a rod with a length $l$ between $4 \mathrm{~mm}$ and $1500 \mathrm{~mm}$ and hemispherical ends with a constant radius of $R=8.8$ $\mathrm{mm}$ were simulated. The initial striking velocity was set to $4 \mathrm{~m} / \mathrm{s}$, and the variations in the energy, vertical velocity, and vertical displacement for rods with lengths of $1500 \mathrm{~mm}$ and $4 \mathrm{~mm}$ are shown in Fig. 3. Once the 1500-mm-long rod contacted the rigid flat surface, the velocity of the hemispherical contact point rapidly dropped to zero and reversed at approximately $0.75 \mathrm{~ms}$, the velocity of the bottom point of the rod reversed at approximately $0.65 \mathrm{~ms}$, and the center and top points reversed earlier than the other two sections, as illustrated in Fig. 3b.

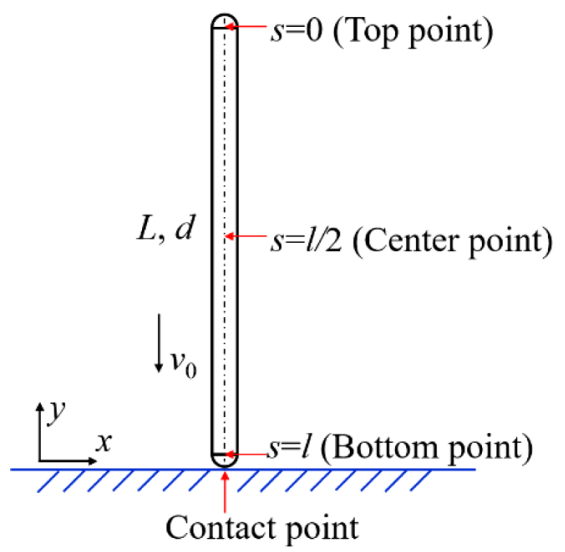

Fig. 1. Schematic diagram of the vertical rod impact. 


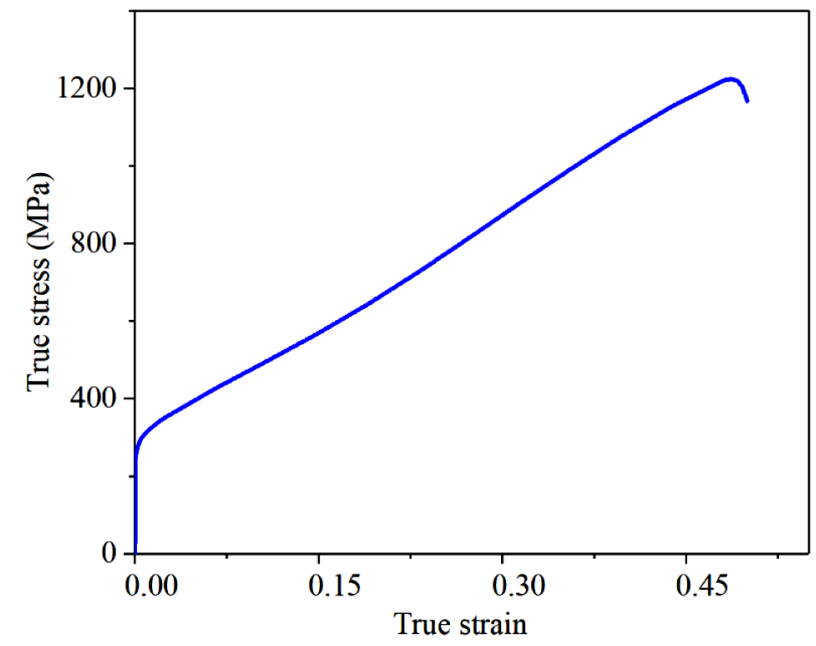

Fig. 2. True stress versus true strain for SS316.

During the entire impact, the kinetic energy decreased with an increase in strain energy followed by the accumulation of plastic dissipation. The strain energy was observed to be largest when the velocity at the top point reversed (i.e., $v_{\text {Top }}=0$ ). After this point, the strain energy decreased with an increase in kinetic energy. When the contact point separated from the rigid flat surface, the dissipated plastic energy reached a maximum. A comparison of Fig. 3b (1500-mm-long rod) and Fig. 3e (4-mm-long rod) showed that the velocity of the $1500-\mathrm{mm}$-long rod was different than that of the 4-mm-long rod, where the velocity of all points for the 4-mm-long rod reversed at the same time. Additionally, the 4-mm-long rod only exhibited a small amount of strain

(a)

(b)

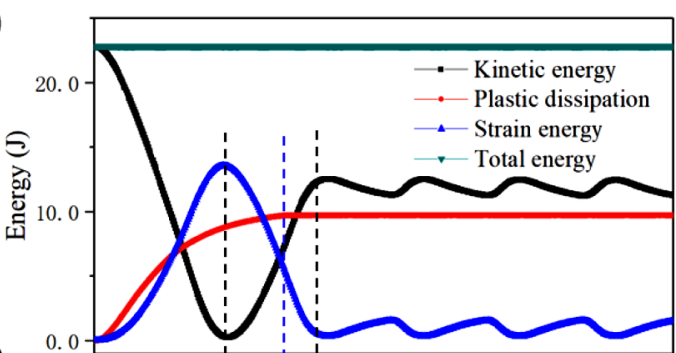

(c)

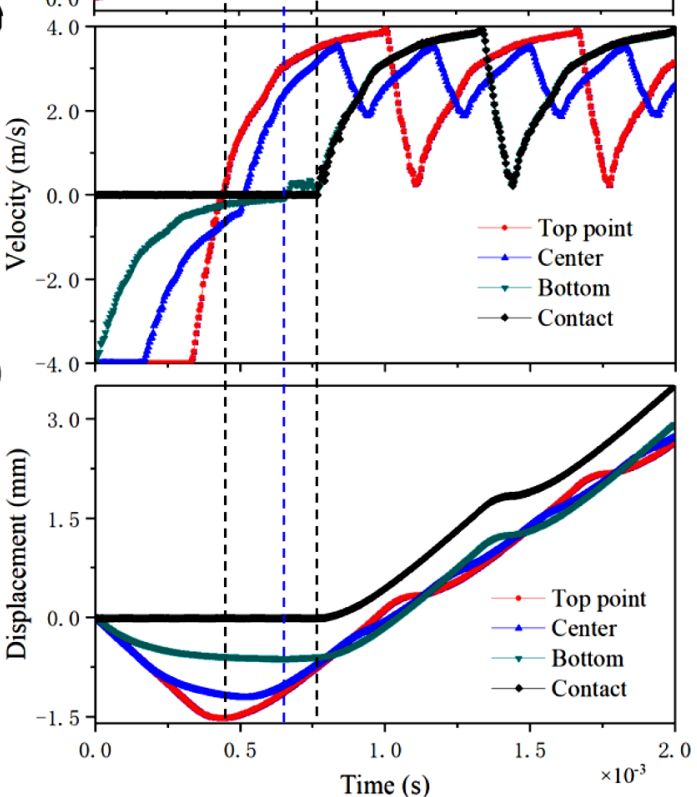

energy after impact, and more than $70 \%$ of the energy was dissipated by plastic deformation, as illustrated in Fig. $3 \mathrm{~d}$. In contrast, the longer rod exhibited a smaller portion of irreversible plastic deformation but a much larger amount of recoverable strain energy.

When the contact points separated from the rigid flat surface, plastic strain in the rods reached a maximum, and its distribution for four different rod lengths is shown in Fig. 4. Here, the plastic deformation was concentrated in the hemispherical section of the rods, as indicated by the gray areas corresponding to equivalent plastic strains greater than $0.06 \%$. Therefore, for a striking velocity of $4 \mathrm{~m} / \mathrm{s}$, the plastic region only occurred in the hemispherical region, and the rest of the rod deformed elastically. The relationship between the impact force and the displacement of the hemispherical tip is illustrated in Fig. 5. The force was essentially linear with respect to the displacement and can be fitted with a slope of $k=(5.61 \pm 0.04) \times 10^{4} \mathrm{~N} / \mathrm{mm}$.

\subsubsection{Equivalent spring model for the contact of the hemispherical tip}

In this subsection, we compared the contact stiffness of the previous dynamic test with the value of the static flattening test, in which the quasistatic loading and unloading of a hemispherical tip with a radius of $8.8 \mathrm{~mm}$ were simulated (the model and corresponding details are shown in Fig. A.1). According to the displacement amplitude from the dynamic simulation in Section 2.2.1, we applied a interference of $0.1-0.617 \mathrm{~mm}$ in this static simulation. To further check the model in the nonlinear region, we also simulated the flattening of a hemisphere with a smaller interference of $0.05 \mathrm{~mm}$. For a linear hardening material such as SS316, the static flattening simulations showed that the contact force $(F)$ versus interference $(\delta)$ curves also had a linear relationship in the interference range of $0.1 \mathrm{~mm}$ to $0.617 \mathrm{~mm}$ (Fig. 6). The difference in equivalent stiffness $k$ between the dynamic impact and static flattening model was less than $2.0 \%$. From this comparison, we can

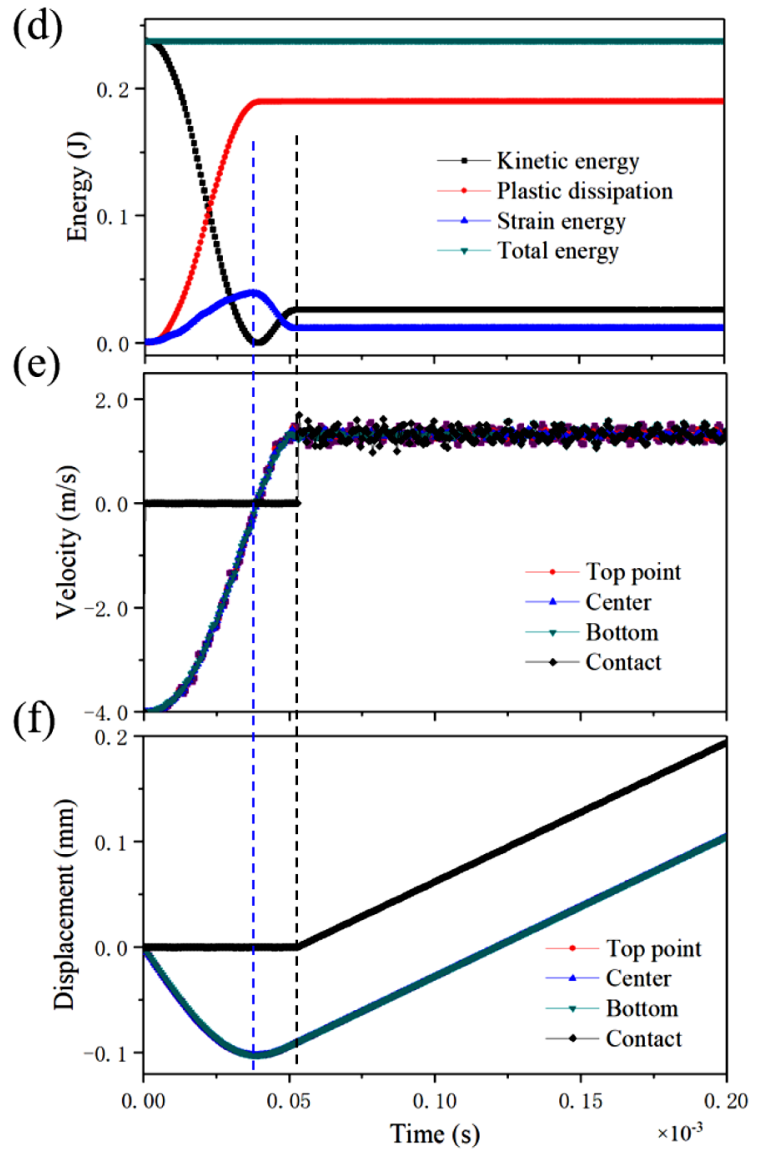

Fig. 3. Variation in energy, vertical velocity, and displacement during the impact for rods with lengths of (a, b, c) $1500 \mathrm{~mm}$ and (d, e, f) $4 \mathrm{~mm}$. 
(a) PEEQ \begin{tabular}{l} 
(Avg: $75 \%$ ) \\
\begin{tabular}{|r|}
$+6.9 \mathrm{e}-02$ \\
$+6.0 \mathrm{e}-04$ \\
$+5.0 \mathrm{e}-04$ \\
$+4.0 \mathrm{e}-04$ \\
$+3.0 \mathrm{e}-04$ \\
$+2.0 \mathrm{e}-04$ \\
$+1.0 \mathrm{e}-04$ \\
$+0.0 \mathrm{e}+00$
\end{tabular} \\
\hline
\end{tabular}

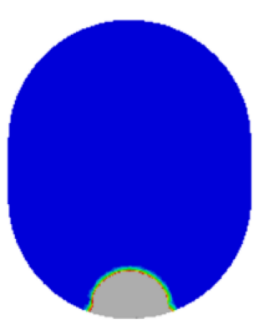

(c) PEEQ

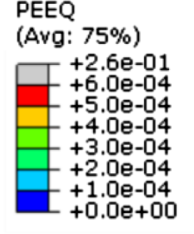

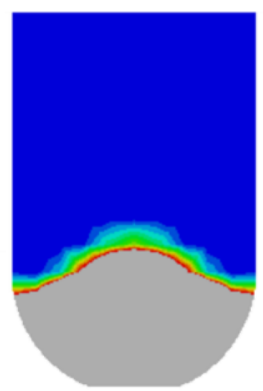

(b)
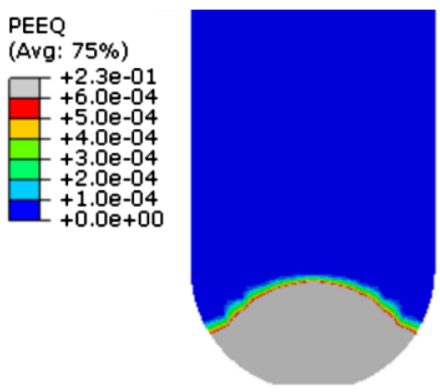

(d)

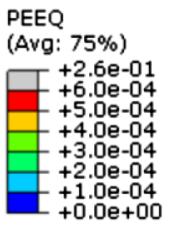

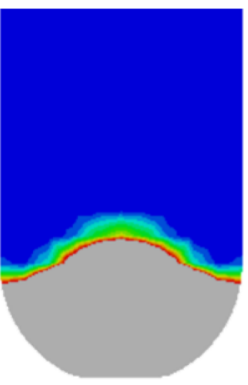

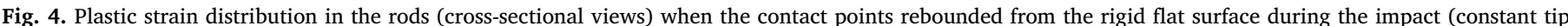
radius $=8.8 \mathrm{~mm}$ ): (a) $l=4 \mathrm{~mm}$, (b) $l=300 \mathrm{~mm}$, (c) $l=1000 \mathrm{~mm}$, and (d) $l=1500 \mathrm{~mm}$.

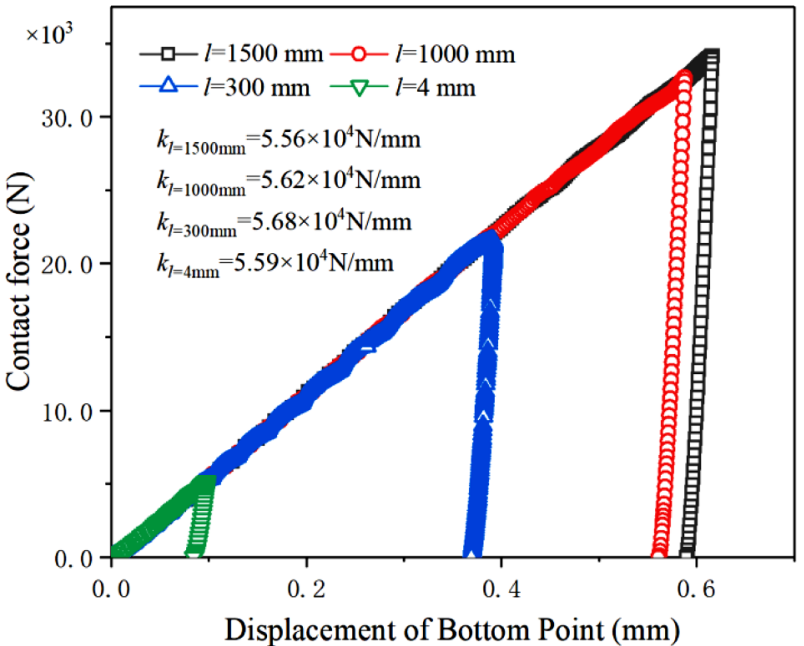

Fig. 5. Relationship between the dynamic contact force and the displacement of the bottom point for rods with lengths of 4-1500 mm.

conclude that the inertial effects can be neglected at these striking velocities so that the quasistatic relationship $F=k \delta$ is applicable for dynamic impacts for striking velocities less than $4 \mathrm{~m} / \mathrm{s}$. In addition, references [32,33] implied that rate sensitivity was negligible for striking velocities of $4 \mathrm{~m} / \mathrm{s}$; hence, in the present model, we neglected the rate sensitivity of the material. However, when the impact velocity was very small and the interference was less than $0.05 \mathrm{~mm}$, the FEM results (the inset in Fig. 6) showed that the KE model should be used because nonlinear behavior occurred. Hence, the present equivalent spring model was not accurate for impacts with very low velocity. Thus, the scope of our model will be discussed in Section 3.6.

Therefore, it is worth discussing the scope of different models. The classic Hertz contact model is only applicable for elastic deformation. In the KE model [24], the hemisphere is fully elastic when $\delta / \delta_{\mathrm{c}} \leq 1$, where $\delta_{\mathrm{c}}$ is the critical interference at which the yield starts. We call this parameter the critical elastic interference $\left(\delta_{\mathrm{c}}\right)$; for example, when the hemisphere has a radius of $8.8 \mathrm{~mm}, \delta_{\mathrm{c}}=0.108 \mu \mathrm{m}$ and $F_{\mathrm{c}}=0.79 \mathrm{~N}$ (Fig. A.2a). As $\delta$ increases, the KE model implies a power-law

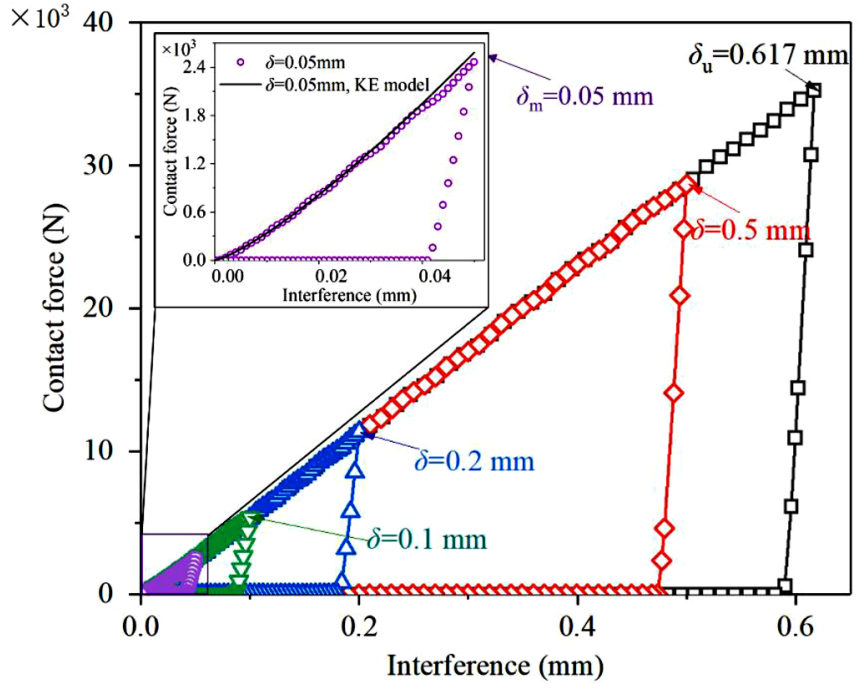

Fig. 6. FEM results for a hemisphere with a radius of $8.8 \mathrm{~mm}$ and an interference of $0.05-0.617 \mathrm{~mm}$. The inset presents a comparison of the FEM results and the KE model results with an interference of $0.05 \mathrm{~mm}$.

relationship for an elastic-perfectly plastic hemisphere [24], wherein $F \propto \delta^{1.425}$ for $1<\delta / \delta_{\mathrm{c}} \leq 6$, and $F \propto \delta^{1.263}$ for $6<\delta / \delta_{\mathrm{c}} \leq 110$, where $110 \delta_{\mathrm{c}} \sim 12 \mu \mathrm{m}$. However, in this study, for a linear hardening hemisphere, we find that when $\delta=50 \mu \mathrm{m}\left(\delta / \delta_{\mathrm{c}} \sim 460\right)$, the contact forceinterference curve still conformed to the relationship $F \propto \delta^{1.263}$, as shown in the inset in Fig. 6 . This means that the use range of the KE model can be extended to $\delta / \delta_{\mathrm{c}}=460$. For clarity, we define it as the maximum nonlinear interference $\left(\delta_{\mathrm{m}}\right)$. Here, it is approximately $460 \delta_{\mathrm{c}}(50 \mu \mathrm{m})$ (Fig. A.2b). When $\delta>\delta_{\mathrm{m}}$, there is a good linear relationship between the contact force and interference $(F \propto \delta)$. Hence, the relationship $F=k \delta$ is suitable for the deep flattening model of SS316, a typical linear hardening material. This linear relationship was also suitable for linear hardening materials with different hardening moduli. When $\delta$ continued to increase until the hemisphere was fully plastic (Fig. A.2c), and we call the corresponding deformation the ultimate interference $\left(\delta_{\mathrm{u}}\right)$.

To verify whether this linear relationship was generally applicable 
for different sizes, the static loading and unloading of the hemispherical tips with radii ranging from $4.4 \mathrm{~mm}$ to $13.2 \mathrm{~mm}$ were also simulated, and the results are shown in Fig. A.3. The quasistatic interferences were taken as a fraction of the tip radius $R$, as illustrated in Fig. A.3a. The equivalent stiffness $k$ was obtained from fitting the linear relationship $F=k \delta$, and values of $k$ are given for four different radii between $4.4 \mathrm{~mm}$ and $13.2 \mathrm{~mm}$ : (1) $R_{1}=4.4 \mathrm{~mm}$ and $k_{1}=28485 \mathrm{~N} / \mathrm{mm}$, (2) $R_{2}=6.6 \mathrm{~mm}$ and $k_{2}=42751 \mathrm{~N} / \mathrm{mm}$, (3) $R_{3}=8.8 \mathrm{~mm}$ and $k_{3}=57095 \mathrm{~N} / \mathrm{mm}$, and (4) $R_{4}=13.2 \mathrm{~mm}$ and $k_{4}=86146 \mathrm{~N} / \mathrm{mm}$. The results show that the hemisphere stiffness and radius also exhibited good linearity (Fig. A.3b), and the stiffness versus radius can be fit to $k=K R$, where $K$ is the equivalent modulus of $6555.3 \mathrm{MPa}$ for SS316.

\subsubsection{Vibration theory of a slender rod with the proposed contact model}

Section 2.2.1 indicates that the straight part of the rod was elastic during the vertical impact with an initial striking velocity of $4 \mathrm{~m} / \mathrm{s}$. Thus, based on Fig. A.4, a theoretical model for the vertical impact of an elastic rod was proposed. The longitudinal motion of a rod $u(s, t)$ is governed by the one-dimensional wave equation, which is expressed as

$\frac{\partial^{2} u}{\partial t^{2}}=c^{2} \frac{\partial^{2} u}{\partial s^{2}}$

where $c$ is the one-dimensional longitudinal wave velocity in the rod and $t$ is time. From Fig. A.4, the boundary and initial conditions are given as follows:

1) The force boundary condition at $s=0$ is expressed as

$$
\left(\frac{\partial u}{\partial s}\right)_{s=0}=0
$$

2) The contact boundary condition at $s=l$ is expressed as

$$
E A\left(\frac{\partial u}{\partial s}\right)_{s=l}=-F_{\mathrm{e}}
$$

where $F_{\mathrm{e}}=k u(l, t)=K R u(l, t), E$ is the Young's modulus of the rod, and $A$ is the cross-sectional area of the rod, which has a value of $\pi R^{2}$.

1) The initial displacement condition is expressed as

$$
u(s, 0)=0
$$

2) The initial velocity condition is expressed as

$$
\left(\frac{\partial u}{\partial t}\right)_{t=0}=v_{0}
$$

By considering the free vibration of an elastic rod as discussed in references $[34,35]$, the displacement field of the rod can be decomposed through the separation of variables.

$u(s, t)=U(s) q(t)$

After substituting Eq. (6) in the one-dimensional wave equation (Eq. (1)), solving for $U(s)$ and $q(t)$ gives the general solution, which can be expressed as

$U(s)=a_{1} \cos \frac{\omega}{c} s+a_{2} \sin \frac{\omega}{c} s$

$q(t)=b_{1} \cos \omega t+b_{2} \sin \omega t$

where $\omega$ is the natural frequency of the rod. In Eq. (7), $a_{1}, a_{2}, b_{1}$, and $b_{2}$ are constants that depend on the boundary and initial conditions.

The boundary condition given by Eq. (2) gives $a_{2}=0$, whereas the boundary condition given by Eq. (3) gives

$\frac{\omega}{c} l \tan \frac{\omega}{c} l=\frac{K R}{E A / l}$

If $\beta=\frac{\omega}{c} l$, Eq. (8) can be reduced to

$\beta \tan \beta=\frac{2 K}{\pi E} \frac{l}{d}$

which depends on the rod aspect ratio $l / d$.

Additionally, because the trigonometric solutions are periodic Eq. (8) satisfies $\forall \omega_{n}=\beta_{n} c / l, n=1,2,3 \cdots \infty$, from which the displacement is given by Eq. (10).

$u(s, t)=\sum_{n=1}^{\infty} a_{1 n} \cos \frac{\omega_{n}}{c} s\left(b_{1 n} \cos \omega_{n} t+b_{2 n} \sin \omega_{n} t\right)$

The $\beta_{n}$ terms are obtained numerically from Eq. (9). The initial conditions for the problem are given by Eqs. (4) and (5). As shown in Eq. (4), $b_{1 n}=0$, and Eq. (11) is obtained from Eqs. (5) and (10).

$\sum_{n=1}^{\infty} a_{1 n} b_{2 n} \omega_{n} \cos \frac{\omega_{n}}{c} s=\sum_{n=1}^{\infty} c_{n} \omega_{n} \cos \frac{\omega_{n}}{c} s=v_{0}$

where the constant $c_{n}=a_{1 n} b_{2 n}$. The orthogonality condition of the eigenfunctions is expressed as

$\int_{0}^{l} \cos \frac{\omega_{n} S}{c} \cos \frac{\omega_{m} S}{c} d s=0, m \neq n, m, n$ (modenumber) $=1,2,3, \ldots$

We integrated the left and right ends of Eq. (11) from 0 to $l$, and values for the constant $c_{n}$ are obtained as

$c_{n}=\frac{v_{0} l \sin \beta_{n}}{c \beta_{n}^{2} \int_{0}^{1}\left(\cos \beta_{n} s\right)^{2} d s}=\frac{2 v_{0} l \sin \beta_{n}}{c \beta_{n}\left(\beta_{n}+\sin \beta_{n} \cos \beta_{n}\right)}$

Using the results from Eq. (13) in Eq. (10) gives the vertical displacement at any point in the rod (determined with Eq. (14)), and differentiating Eq. (14) gives the values for the vertical velocity at any point in the rod (determined with Eq. (15)). (a)

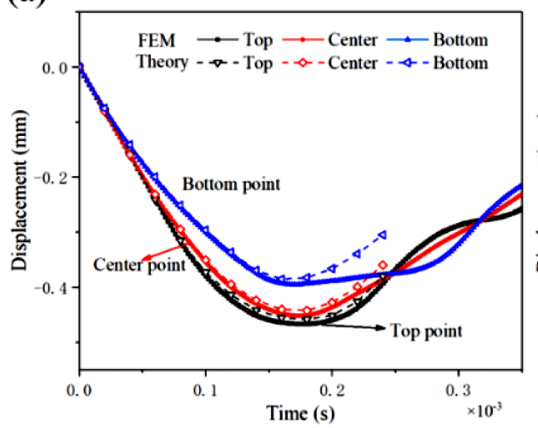

(b)

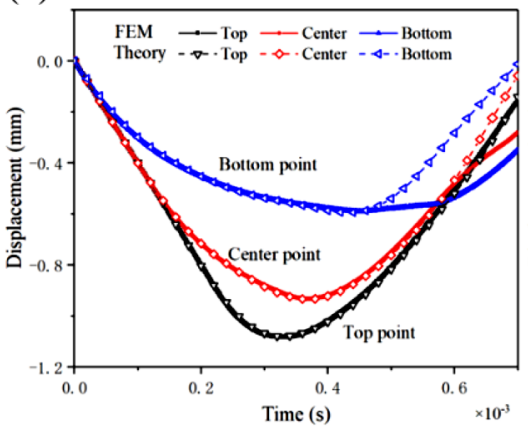

(c)

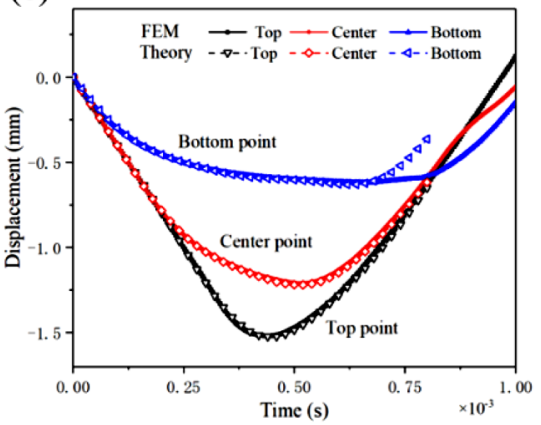

Fig. 7. Vertical displacement during the impact: (a) $l=300 \mathrm{~mm}$, (b) $l=1000 \mathrm{~mm}$, and (c) $l=1500 \mathrm{~mm}$. 
(a)

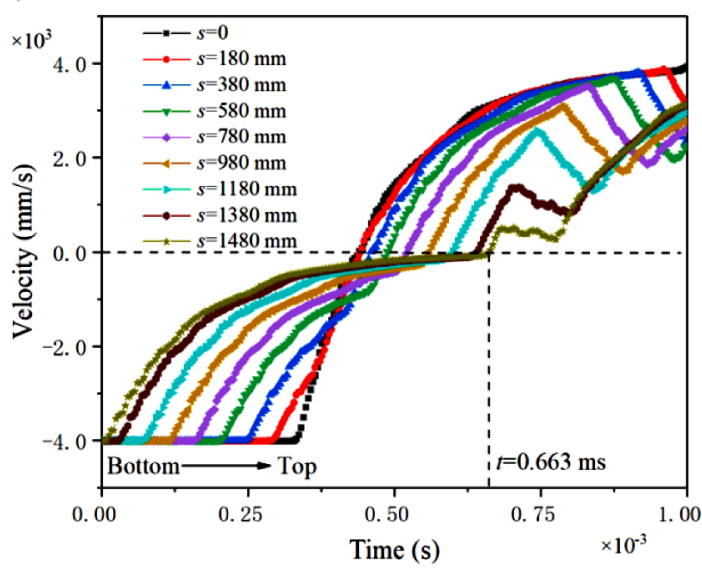

(b)

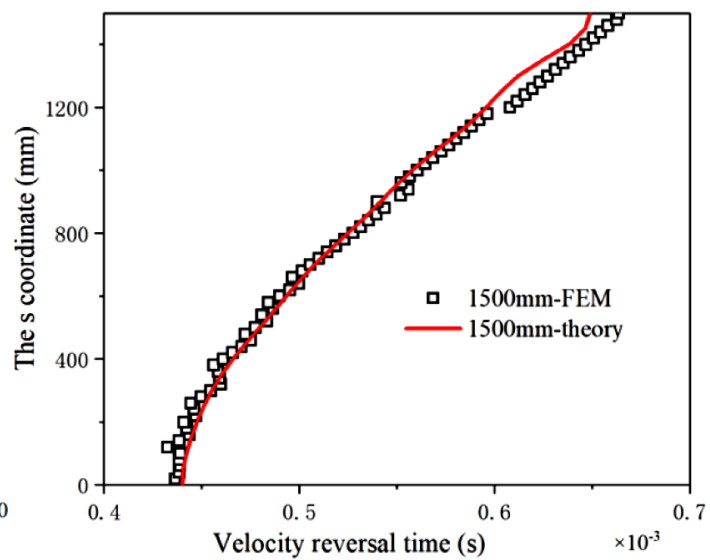

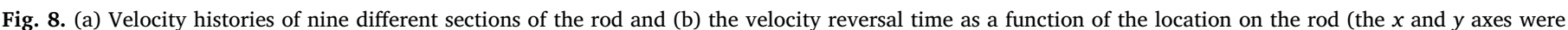
reversed to maintain consistency with Fig. 9).

(a)

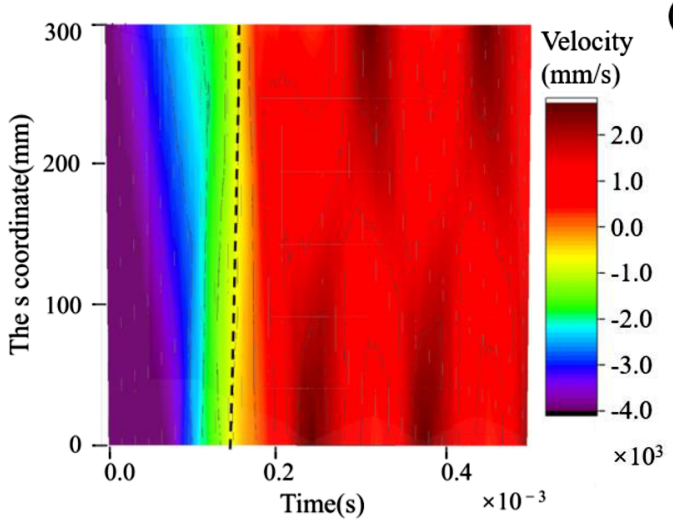

(c)

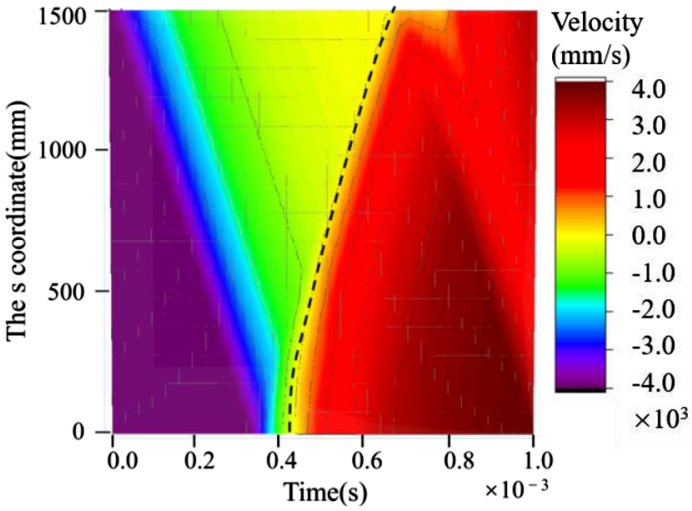

(b)

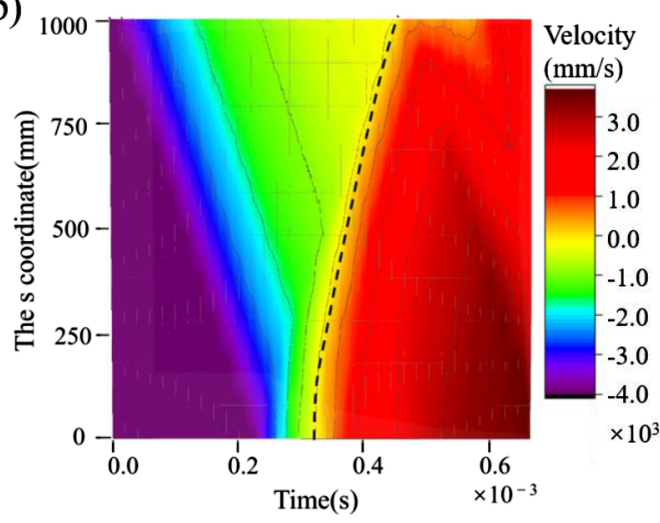

Fig. 9. Vertical velocity during the dynamic impact: (a) $l=300 \mathrm{~mm}$, (b) $l=1000 \mathrm{~mm}$, and (c) $l=1500 \mathrm{~mm}$.

$u(s, t)=\sum_{n=1}^{\infty} \frac{2 v_{0} l \sin \beta_{n}}{c \beta_{n}\left(\beta_{n}+\sin \beta_{n} \cos \beta_{n}\right)} \cos \left(\beta_{n} \frac{s}{l}\right) \sin \left(\omega_{n} t\right)$

$v(s, t)=\frac{\partial u(s, t)}{\partial t}=\sum_{n=1}^{\infty} \frac{2 v_{0} \sin \beta_{n}}{\beta_{n}+\sin \beta_{n} \cos \beta_{n}} \cos \left(\beta_{n} \frac{s}{l}\right) \cos \left(c \beta_{n} \frac{t}{l}\right)$

For the problems considered in this study, we examined the number of terms required in Eqs. (14) and (15) for the displacement and velocity of the rod, as discussed in Appendix A, and found that accurate results were obtained by retaining only the first two terms of the corresponding infinite series, as given by Eqs. (16) and (17).

$$
\begin{aligned}
u(s, t) & =\frac{2 v_{0} l \sin \beta_{1}}{c \beta_{1}\left(\beta_{1}+\sin \beta_{1} \cos \beta_{1}\right)} \cos \left(\beta_{1} \frac{s}{l}\right) \sin \left(c \beta_{1} \frac{t}{l}\right) \\
& +\frac{2 v_{0} l \sin \beta_{2}}{c \beta_{2}\left(\beta_{2}+\sin \beta_{2} \cos \beta_{2}\right)} \cos \left(\beta_{2} \frac{s}{l}\right) \sin \left(c \beta_{2} \frac{t}{l}\right) \\
v(s, t) & =\frac{2 v_{0} \sin \beta_{1}}{\beta_{1}+\sin \beta_{1} \cos \beta_{1}} \cos \left(\beta_{1} \frac{s}{l}\right) \cos \left(c \beta_{1} \frac{t}{l}\right) \\
& +\frac{2 v_{0} \sin \beta_{2}}{\beta_{2}+\sin \beta_{2} \cos \beta_{2}} \cos \left(\beta_{2} \frac{s}{l}\right) \cos \left(c \beta_{2} \frac{t}{l}\right)
\end{aligned}
$$

where the parameters $\beta_{1}=-0.29+0.3 \ln (l / d+3.12) \quad$ and $\beta_{2}=0.18+0.68 \ln (l / d+74.19)$ are numerically fitted from Eq. (9) in Fig. A.5. For rods with any specific geometry, the history of displacement and velocity can be calculated by Eqs. (16) and (17), respectively. 


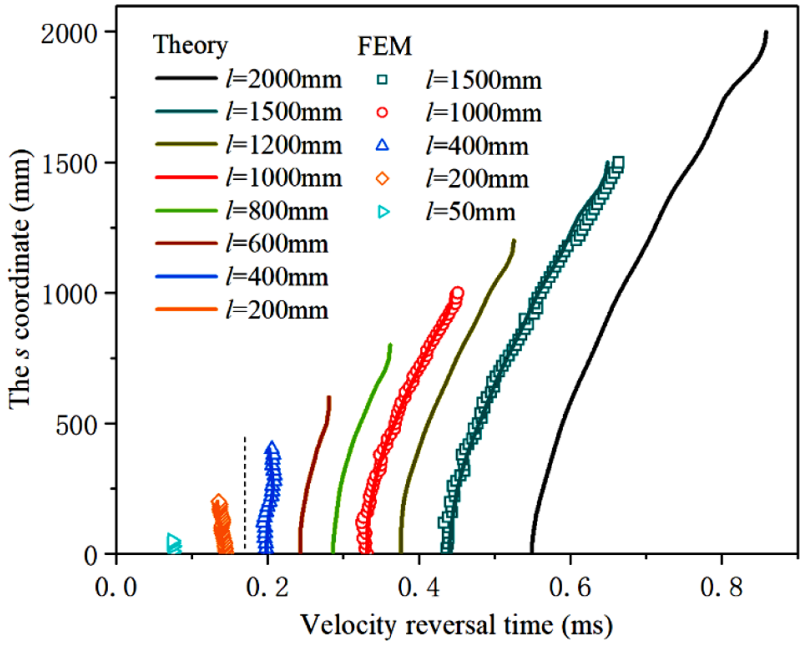

Fig. 10. Velocity reversal time of each point of the rods with lengths of $50-2000 \mathrm{~mm}$ and a diameter of $17.6 \mathrm{~mm}$. The FEM results are represented by open symbols, whereas the theoretical predictions are indicated by lines.

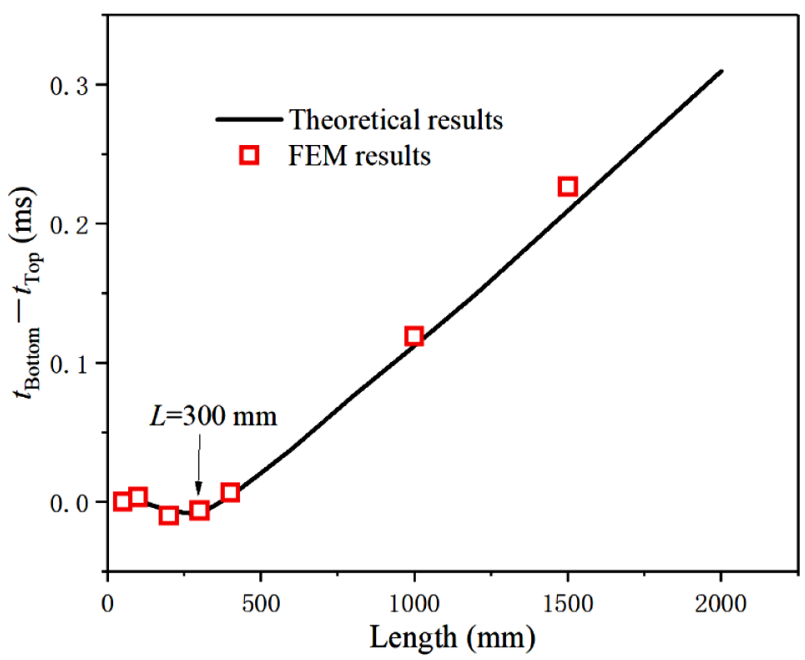

Fig. 11. Difference in velocity reversal time between the bottom and top points for rods with lengths of 50-2000 mm and a diameter of $17.6 \mathrm{~mm}$.

Table 1

$\beta_{1}-\beta_{4}$ and $|\Delta v|_{\max }$ for $l=4 \mathrm{~mm}, 40 \mathrm{~mm}, 400 \mathrm{~mm}$, and $4000 \mathrm{~mm}$.

\begin{tabular}{lllll}
\hline Rod length $(\mathrm{mm})$ & 4 & 40 & 400 & 4000 \\
\hline$\beta_{1}$ & 0.07449 & 0.23361 & 0.68309 & 1.33509 \\
$\beta_{2}$ & 3.14336 & 3.15919 & 3.30808 & 4.07933 \\
$\beta_{3}$ & 6.28407 & 6.29202 & 6.37023 & 6.95733 \\
$\beta_{4}$ & 9.42537 & 9.43067 & 9.48333 & 9.93492 \\
$|\Delta v|_{\max }$ & 0.02109 & 0.20825 & 1.82315 & 6.92247 \\
\hline
\end{tabular}

\section{Comparison of the theoretical model and numerical FEM simulations}

\subsection{Vertical displacement of the points on the rod}

Variations in the vertical displacements at the top, center, and bottom points of the rod during impact for rods with lengths of $l=300$ $\mathrm{mm}, l=1000 \mathrm{~mm}$, and $l=1500 \mathrm{~mm}$ are shown in Fig. 7 . For the theoretical displacement curves, the approximate solution from Eq. (16) was used. Fig. 7 shows that the theoretical model agreed well with the FEM simulations. In all cases, the maximum displacements of the top and center points were greater than that of the bottom point. The longer rods exhibited greater displacements in each of the three sections. Additionally, the time required for each section to reach its maximum displacement was different for each of the different rod lengths, and the top, center and bottom sections reached their maximum displacement in sequence.

\subsection{Variation in the vertical velocity of the rods}

Fig. 8a shows the FEM results for the velocity in nine different sections of a 1500-mm-long rod. The velocity reversal time at any location was obtained from its intersection with the point at which the velocity $v=0$. For the section where $s=1480 \mathrm{~mm}$, the corresponding velocity reversal time was at $t=0.663 \mathrm{~ms}$. Velocity reversal times for all of the other sections were determined in the same way. Additionally, the relationship between the location $s$ of the rod and the reversal time is shown in Fig. 8b using both the FEM result and the theoretical model. Based on Eq. (17), the theoretical reversal time was obtained numerically by solving the implicit equation given by

$v(s, t)=0$

where $s$ varied between 0 and $1500 \mathrm{~mm}$ for the 1500-mm-long rod. As illustrated in Fig. 8b, the theoretical prediction from Eq. (18) agreed well with the FEM results.

The evolution of the rod velocity is also illustrated in Fig. 9. In Fig. 9, the abscissa represents the time, whereas the ordinate represents the location on the rod. In Figs. 8a through c, the color of the cloud maps represents the magnitude of the velocity for rods with lengths $l$ of $300 \mathrm{~mm}, 1000 \mathrm{~mm}$, and $1500 \mathrm{~mm}$. The dashed lines in Fig. 9 show the velocity reversal times required at any location on the rod. For the 300 mm-long rod in Fig. 9a, the dashed line is essentially vertical, which indicates that the velocity reversal time at each point on the rod is approximately constant. For the other two rod lengths, as shown in Figs. $9 \mathrm{~b}$ and c, the velocity reversal time gradually increased from the top point to the bottom point. Thus, the top points reversed first and then turned towards the bottom points. Additionally, the dashed line in Fig. 9c was the same as the curve in Fig. $8 \mathrm{~b}$, which implied that the proposed theoretical model can be used to predict the evolution of the velocity during impact.

\subsection{Velocity reversal time at each point of the rod}

As shown in Fig. 9, for rods with lengths $l$ of $300 \mathrm{~mm}, 1000 \mathrm{~mm}$, and $1500 \mathrm{~mm}$ and a diameter $d=17.6 \mathrm{~mm}$, different trends were observed for the different rod lengths. In this subsection, rods with other lengths are also examined. Fig. 10 shows typical FEM results and theoretical predictions from Eq. (18) for rods with lengths between $50 \mathrm{~mm}$ and $2000 \mathrm{~mm}$

The results showed that when the rod length was greater than 300 $\mathrm{mm}$, the trend of the $s$ versus velocity reversal time curves was similar, and the top point reversed earlier than the bottom point. When the rod length was less than $300 \mathrm{~mm}$, the $s$ versus velocity reversal time curves were nearly vertical, and the bottom and top points reversed simultaneously. This implied the existence of a critical length that was approximately $l_{\mathrm{cr}}=300 \mathrm{~mm}$, giving a critical aspect ratio of $l_{\mathrm{cr}} / d=17.05$ for $d=17.6 \mathrm{~mm}$. Thus, the vibration effect must be considered for $l_{\mathrm{cr}} / d$ $>17.05$, whereas for $l_{\mathrm{cr}} / d<17.05$, the rod can be modeled as a rigid body.

This critical aspect ratio was also applicable for rods with other diameters. The $\beta_{n}$ terms from Eq. (9) were determined by two factors: $2 K /(\pi E)$, which was related to the material properties, and the aspect ratio $l / d$, which was related to the geometric configuration. The velocity reversal time for any point on the rods can be calculated by setting the right side of Eq. (15) to zero. By analyzing the right side of Eq. (15), we find that for cases with the same initial striking velocity $v_{0}$ and the same $\beta_{n}$, the reversal time $t / l$ depends solely on the relative position $s / l$. Furthermore, if we keep $2 K /(\pi E)$ and $l / d$ unchanged, the reversal time 
(a)

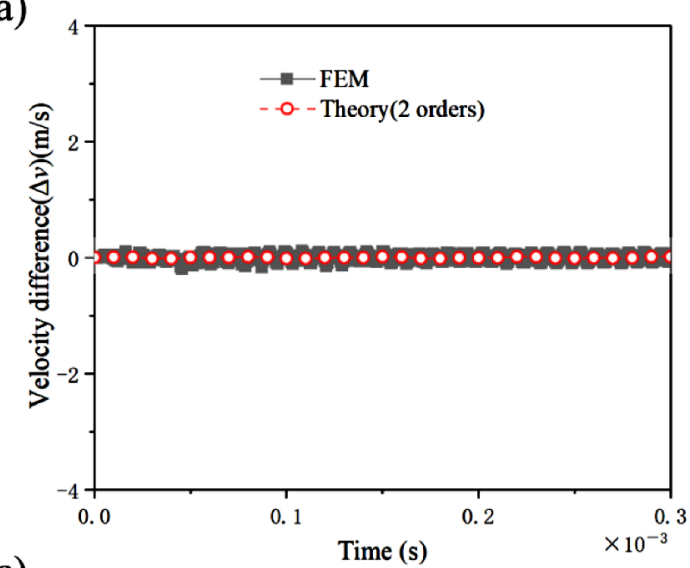

(c)

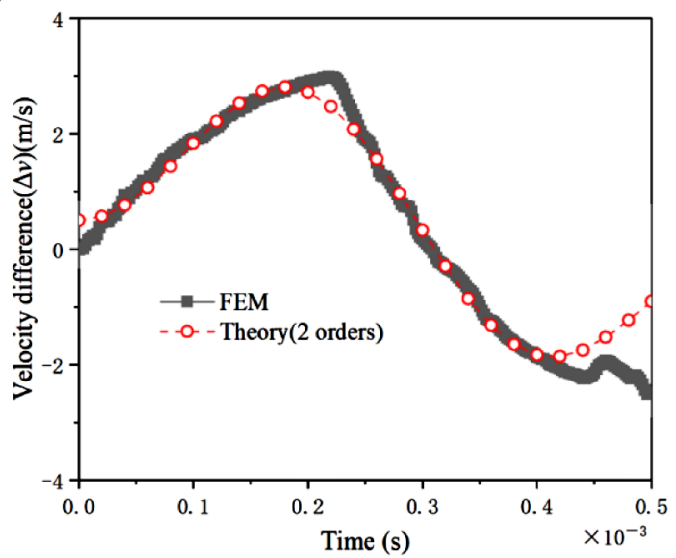

(b)

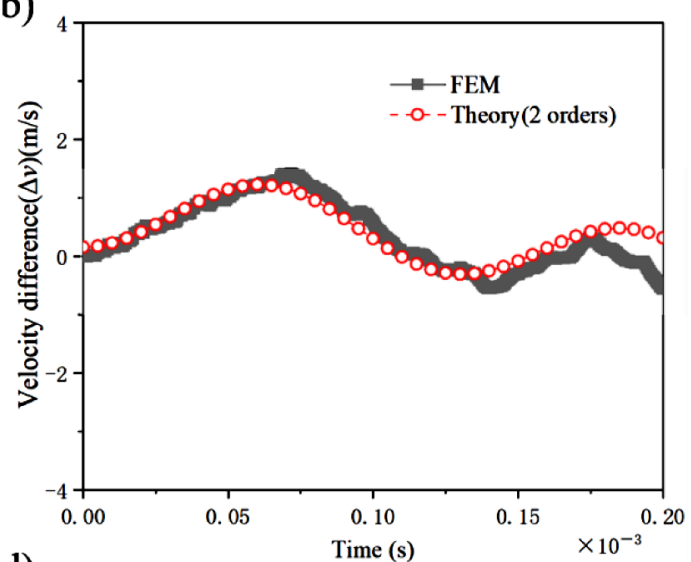

(d)

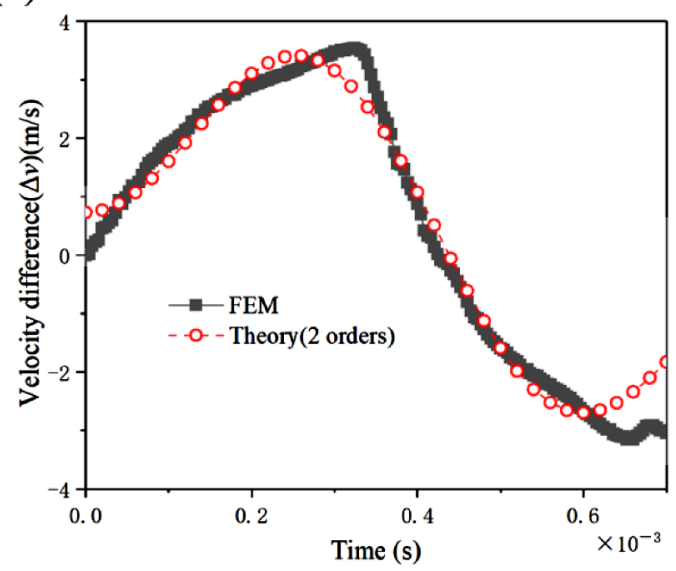

Fig. 12. Vertical velocity difference between the top and bottom points: (a) $l=4 \mathrm{~mm}$, (b) $l=300 \mathrm{~mm}$, (c) $l=1000 \mathrm{~mm}$, and (d) $l=1500 \mathrm{~mm}$.

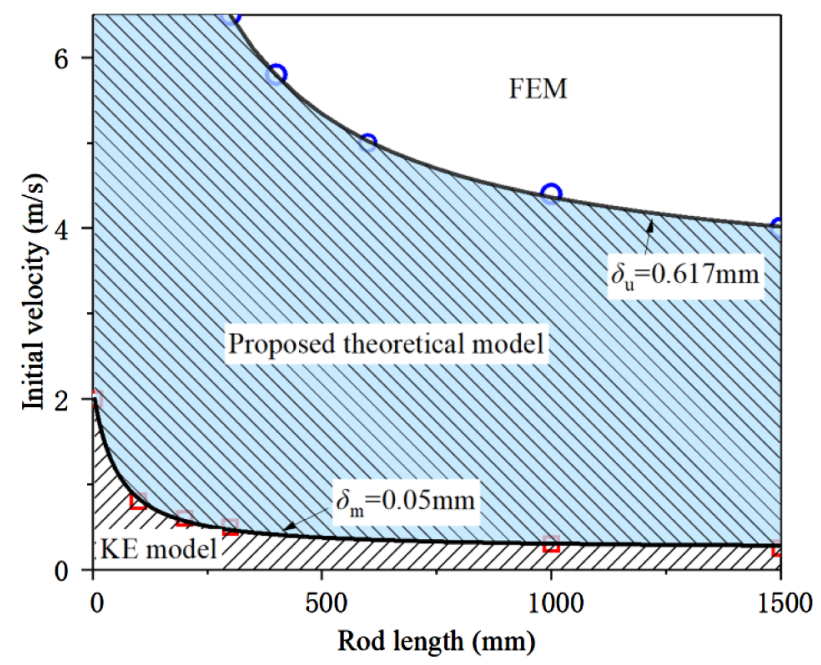

Fig. 13. Application region of the present model for the SS316 rod with a tip radius of $8.8 \mathrm{~mm}$. The blue circle and red square represent the upper and lower boundaries in the FEM simulations, respectively.

of the same relative position is proportional to the rod length such that $t \propto l$. Thus, as obtained from the previous paragraph, the critical aspect ratio $(l / d)_{\text {cr }}$ of 17.05 can be used for rods with different diameters. When $(l / d)_{\text {cr }}$ was larger than 17.05 , the vibration of the elastic part of the rod must be considered. This conclusion was verified from four sets of rods with different aspect ratios $(l / d=11.36,17.05,22.73$, and 34.09) using the FEM, as shown in Fig. A.6.

\subsection{Velocity reversal time difference between the bottom and top points}

By solving the implicit relation in Eq. (18), the theoretical difference in the velocity reversal time between the bottom and top points $t_{\text {Bottom }}{ }^{-}$ $t_{\text {Top }}$ for different rods can be obtained. Considering rods with a diameter of $17.6 \mathrm{~mm}$ as an example, the theoretical relationship between $t_{\mathrm{Bottom}^{-}}$ $t_{\text {Top }}$ and the rod length is plotted in Fig. 11. The results show that the theoretical prediction agreed well with the FEM results. For $l>300 \mathrm{~mm}$ $(l / d>17.05), t_{\text {Bottom }} t_{\text {Top }}$ was proportional to the rod length, whereas for $l<300 \mathrm{~mm}(l / d<17.05), t_{\text {Bottom }} t_{\text {Top }}$ was essentially independent of the rod length and remained near zero.

\subsection{Velocity difference between the top and bottom points}

The velocity difference between the top $(s=0)$ and bottom $(s=l)$ points is considered and theoretically given by

$\Delta v=v(0, t)-v(l, t)$

$=\sum_{n=1}^{\infty} \frac{2 v_{0} \sin \beta_{n}}{\beta_{n}+\sin \beta_{n} \cos \beta_{n}}\left(1-\cos \beta_{n}\right) \cos \frac{c \beta_{n} t}{l}$

$=\sum_{n=1}^{\infty} f\left(\beta_{n}\right) \cos \frac{c \beta_{n} t}{l}$

$=f\left(\beta_{1}\right) \cos \frac{c \beta_{1} t}{l}+f\left(\beta_{2}\right) \cos \frac{c \beta_{2} t}{l}+f\left(\beta_{3}\right) \cos \frac{c \beta_{3} t}{l}+\ldots$ 
where the coefficient $f(\beta)$ is given by

$f(\beta)=\frac{2 v_{0} \sin \beta}{\beta+\sin \beta \cos \beta}(1-\cos \beta)$

Considering rods with lengths $l$ of $4 \mathrm{~mm}, 40 \mathrm{~mm}, 400 \mathrm{~mm}$, and 4000 $\mathrm{mm}$ as examples, their first to fourth orders $\left(\beta_{1}-\beta_{4}\right)$ and $|\Delta v|_{\max }=\left|f\left(\beta_{1}\right)\right|+\left|f\left(\beta_{2}\right)\right|+\left|f\left(\beta_{3}\right)\right|+\left|f\left(\beta_{4}\right)\right|$ are given in Table 1 , and $f$ $(\beta)$ is shown in Fig. A.7. Here, $\left|f\left(\beta_{1}\right)\right|$ and $\left|f\left(\beta_{2}\right)\right|$ are significantly larger than $\left|f\left(\beta_{3}\right)\right|$ and $\left|f\left(\beta_{4}\right)\right|$; therefore, it can be concluded that the first two terms dominate the solution.

For rod lengths $l$ between $4 \mathrm{~mm}$ and $1500 \mathrm{~mm}$, the theoretical predictions containing $\beta_{1}$ and $\beta_{2}$ agreed well with the FEM results, as shown in Fig. 12. We also compared the theoretical predictions with different orders and found that the difference was small, as shown in Fig. A.8, which indicated that only considering the first two orders was sufficient to accurately predict the velocity difference. Hence, Eq. (21) was used to determine the velocity difference between the top and bottom points during impact.

$\Delta v=f\left(\beta_{1}\right) \cos \frac{c \beta_{1} t}{l}+f\left(\beta_{2}\right) \cos \frac{c \beta_{2} t}{l}$

\subsection{Scope of the proposed theoretical model}

The theoretical model proposed in Section 2.2.3 was based on two assumptions, which were the equivalent contact model and that the straight part of the rod was elastic. If the impact velocity was sufficiently low that the impact was purely elastic, then the interference was less than $\delta_{\mathrm{c}}$, and the Hertz contact model was applicable and should be employed. If the impact velocity was intermediate, the impact was elasto-plastic, the interference of the bottom hemisphere was less than $\delta_{\mathrm{m}}$, and the KE model should be employed. For high impact velocities, plasticity was dominant, and the interference of the bottom hemisphere was much larger than $\delta_{\mathrm{m}}$, and the proposed model in this study should be employed for the tip contact to predict the displacement and velocity histories. For this case, the maximum interference $\delta_{\mathrm{m}}$ was related to the initial impact velocity and length of the rod by Eq. (22).

$\frac{1}{2} m v_{0, K E}^{2} \approx \int_{0}^{\delta_{\mathrm{m}}} F d \delta$

where $m$ is the mass of the rod and the force is given by $F=k \delta$. From Eq. (22), for a rod with a length of $l$, the maximum initial impact velocity applicable for the KE model is given by Eq. (23).

$v_{0, K E} \approx \sqrt{\frac{k \delta_{\mathrm{m}}^{2}}{\rho\left(\frac{4}{3} \pi R^{3}+\pi R^{2} l\right)}}$

where $\rho$ is the material density. For the case of a hemisphere with a radius of $8.8 \mathrm{~mm}$, the lower boundary relationship between the striking velocity and rod length is shown in Fig. 13 and can be calculated by Eq. (23), in which $\delta_{\mathrm{m}}=460 \delta_{\text {c. }}$ This lower velocity value in Fig. 13 was also obtained by FEM impact simulations of rods with different combinations of length and striking velocity. The upper boundary of the proposed theoretical model depended on the distribution of plastic deformation in the rod and was determined using FEM simulations under the assumptions that the straight section of the rod was elastic and that plastic deformation only occurred in the bottom hemisphere of the $\operatorname{rod}\left(\delta \leq \delta_{\mathrm{u}}\right.$, where $\delta=\delta_{\mathrm{u}}$ represents the hemisphere entering full plasticity, as shown in Fig. A.2c). Within the blue hatched region, the proposed model and Eqs. (16) and (17) can be used. For long rods, the $\mathrm{KE}$ model will be out of its applicable range, even for varying low striking velocities. For instance, we should use the proposed model for a 1000 -mm-long rod with an initial striking velocity greater than $0.5 \mathrm{~m} / \mathrm{s}$, as shown in Fig. 13.

\section{Conclusions}

A semianalytical model was developed to model the plastic impact while considering both the contact of the rod hemispherical tip and the vibration effects generated in the straight portion of the rod. The major conclusions are as follows:

1) The proposed semianalytical model can predict the vertical impact between a slender SS316 rod and a rigid flat surface before rebounding by Eqs. (16) and (17). During this period, the velocity difference between the top and bottom points can be determined from Eq. (21).

2) For the vertical rod impact, a critical length/diameter aspect ratio was obtained from the proposed theoretical model. The results showed that for $l / d<17$, the rod behaved as a rigid body. For $l /$ $d>17$, the vibration effects were significant, and the velocity of the top section of the rod reversed much earlier than the other sections of the rod.

3) To illustrate the applicable region of the proposed model, a map associated with the initial striking velocity and the length of the rod was established. For SS316 rods with a hemispherical tip radius of $8.8 \mathrm{~mm}$, the proposed model can be applied within the blue hatched region shown in Fig. 13.

\section{CRediT authorship contribution statement}

Xuan Ye: Methodology, Software, Writing - original draft, Formal analysis, Writing - review \& editing. Jianqiao Hu: Validation, Writing review \& editing. Jingru Song: Formal analysis, Writing - review \& editing. Xiaoming Liu: Conceptualization, Project administration, Funding acquisition, Supervision, Writing - review \& editing, Formal analysis. Yueguang Wei: Project administration, Formal analysis, Supervision.

\section{Declaration of Competing Interest}

The authors declare no competing financial interests.

\section{Acknowledgements}

This work was supported by the National Natural Science Foundation of China (no. 11902311, 11972347, 11772334), by China Postdoctoral Science Foundation (no. 2019M650699), by Youth Innovation Promotion Association CAS (2018022), and by the Strategic Priority Research Program of the Chinese Academy of Sciences (no. XDB22040501). We also thank Dr. Wang Tao and Prof. Zhuang Zhuo from Tsinghua University for the help of finite element simulations. 


\section{Appendix A}

The FEM models were established using ABAQUS Standard, wherein C3D8R solid elements were used to mesh the rod. The global mesh size was $0.5 \mathrm{~mm}$, and the mesh size of the hemisphere contact was $0.1 \mathrm{~mm}$. Additionally, the mesh sensitivity of the contact between a hemisphere and a rigid flat surface was checked, and the results were found to be convergent. Eight figures (Figs. A.1-A.8) are provided in this section and are referenced throughout the main sections of the manuscript.

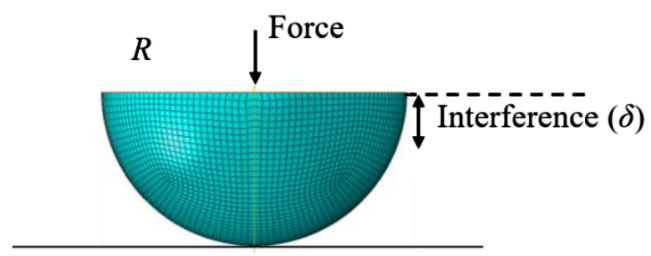

Fig. A.1. FEM model for hemispherical flattening.

(a)

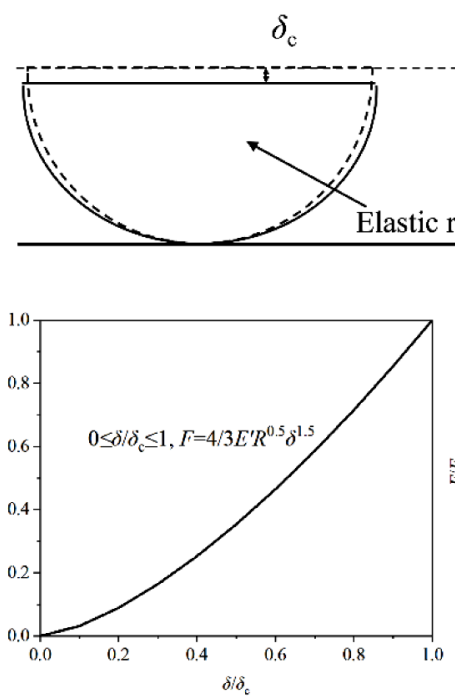

(b)

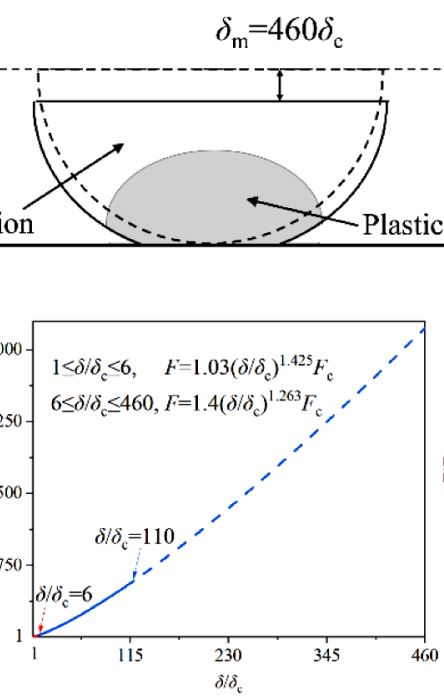

(c)
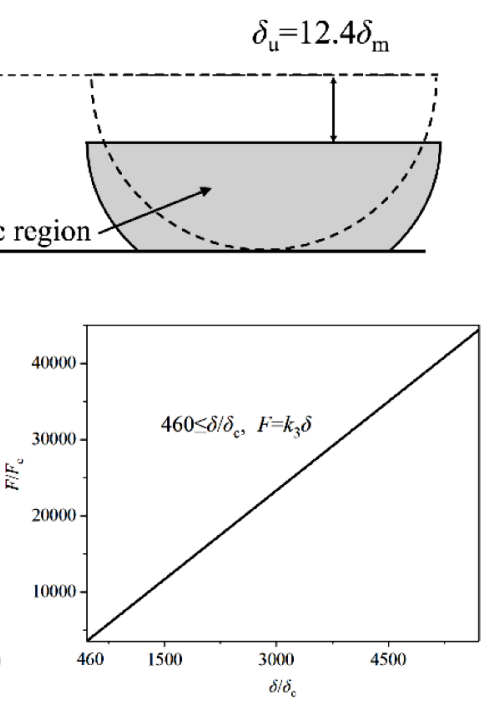

Fig. A.2. Schematic diagram of the loading and deformation of a hemispherical tip. The blank and gray areas represent the elastic and plastic regions, respectively. $E^{\prime}=E /\left(1-v^{2}\right)$, where $\nu$ is the Poisson's ratio. For a hemisphere with a radius of $8.8 \mathrm{~mm}, \delta_{\mathrm{c}}=0.108 \mu \mathrm{m}, F_{\mathrm{c}}=0.79 \mathrm{~N}$, and $k_{3}=57095 \mathrm{~N} / \mathrm{mm}$.

(a)

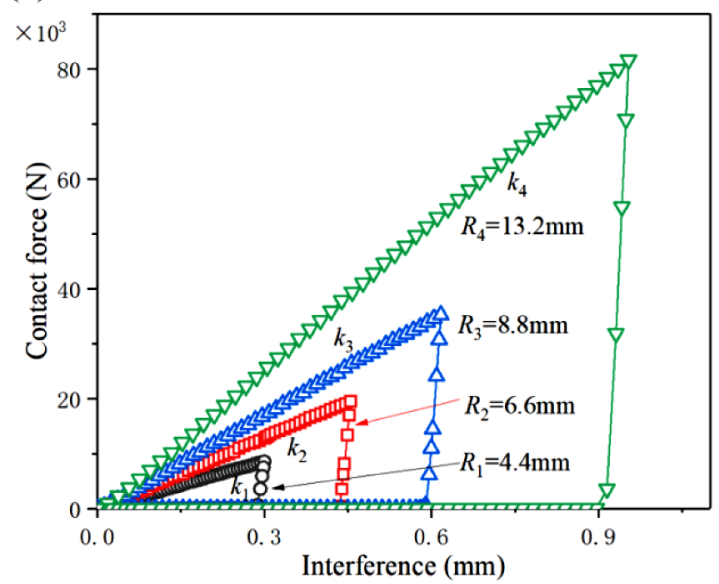

(b)

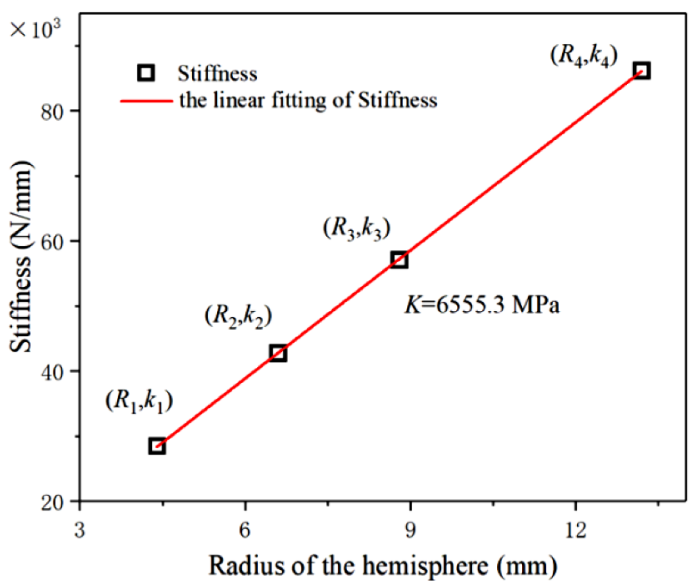

Fig. A.3. (a) Static contact force-interference curves for hemisphere radii of 4.4-13.2 $\mathrm{mm}$. (b) Relationship between the hemisphere stiffness and radius. 


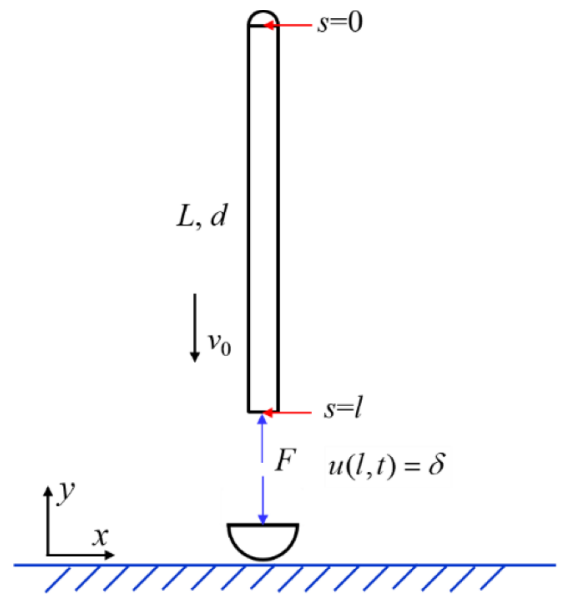

Fig. A.4. Theoretical model for the rod impact.

(a)

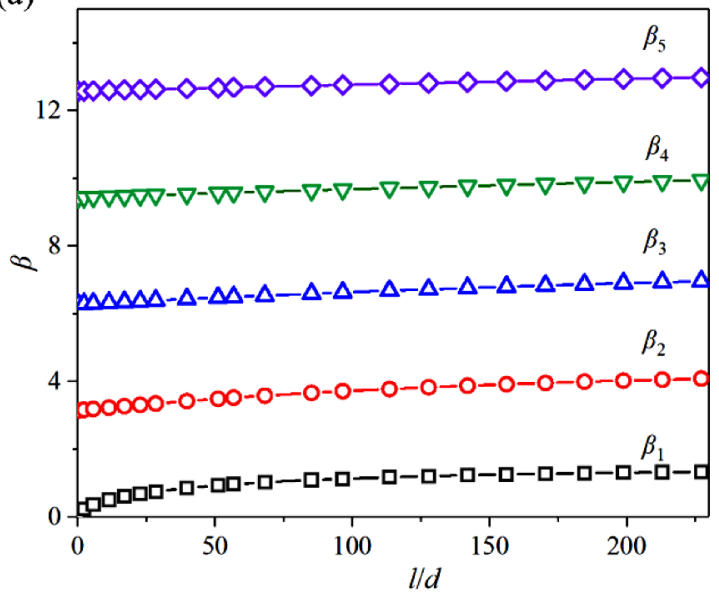

(b)

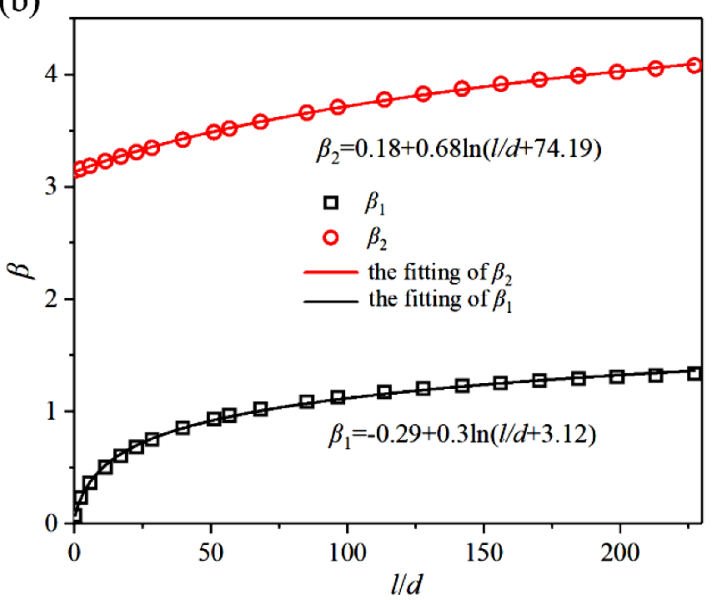

Fig. A.5. Theoretical relationship between $\beta$ and aspect ratio $(l / d)$. (a) Theoretical solutions for $\beta_{1}-\beta_{5}$. (b) Fitting $\beta_{1}$ and $\beta_{2}$ with the rod aspect ratio $(l / d)$.

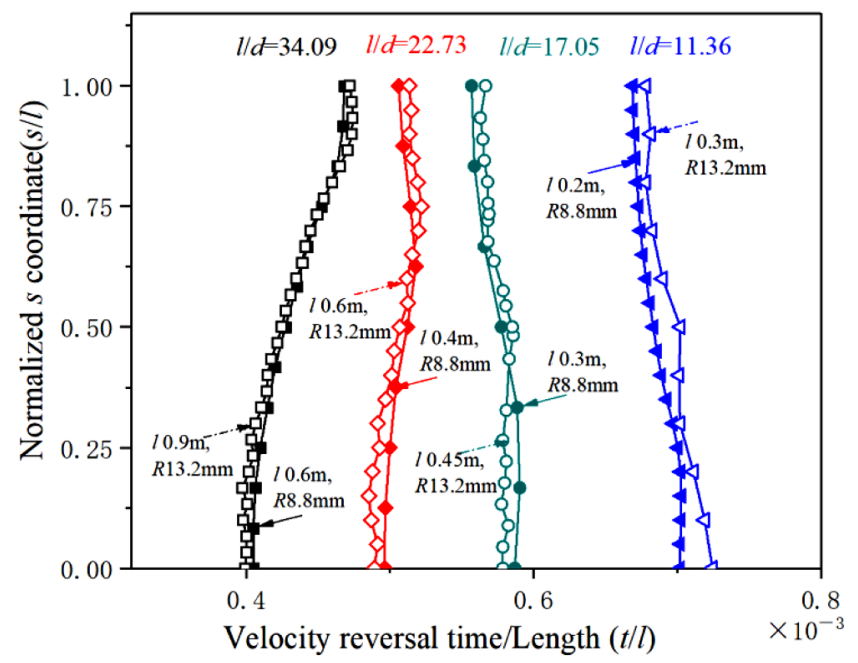

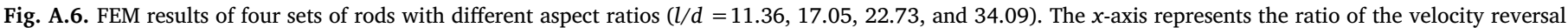
time $(t)$ to the rod length $(l)$. The $y$-axis represents the normalized $s$-coordinate position by rod length $(s / l)$. 


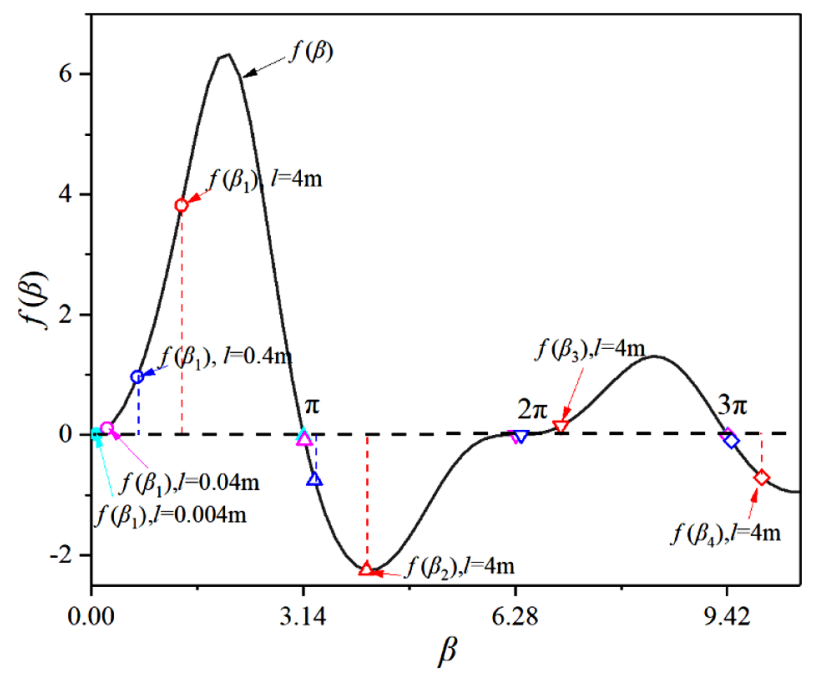

Fig. A.7. Values of $f(\beta)$ for rods with lengths of $0.004-4 \mathrm{~m}$. The circles in the figure represent the $f\left(\beta_{1}\right)$ values of rods with different lengths. The upright triangles, inverted triangles, and diamonds represent $f\left(\beta_{2}\right), f\left(\beta_{3}\right)$, and $f\left(\beta_{4}\right)$ of rods with different lengths, respectively.
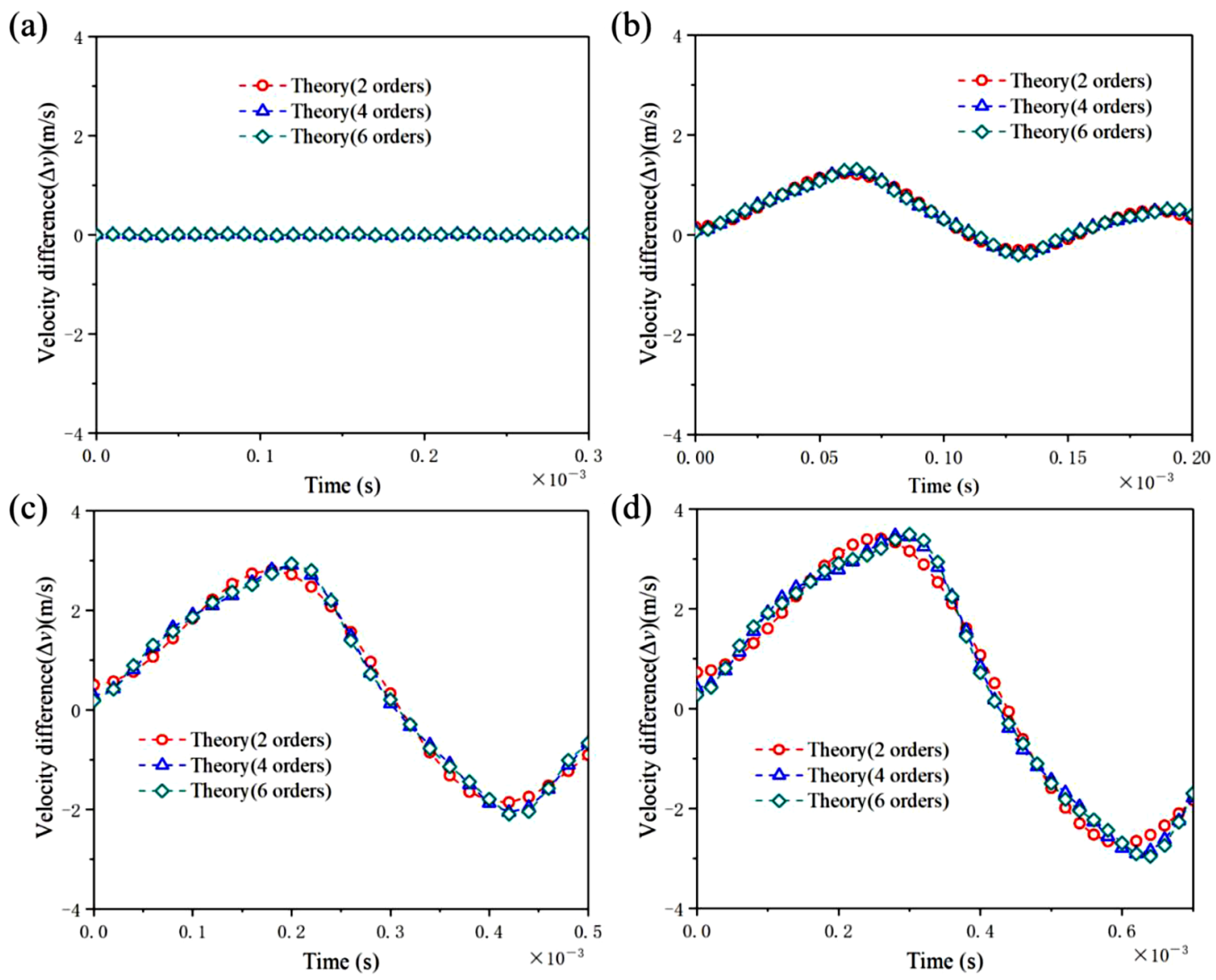

Fig. A.8. Theoretical predictions with different orders for rods with lengths of 0.004-4 m.

\section{References}

[1] Zhang Z, Li L, Zhang D. Effect of arbitrary yaw/pitch angle in bird strike numerical simulation using SPH method. Aerosp Sci Technol 2018;81:284-93. https://doi. org/10.1016/j.ast.2018.08.010.

[2] Dong X, Xiaochun Y, Qingming D, Bo Y, Hui W, Panpan W, et al. Local contact behavior between elastic and elastic-plastic bodies. Int J Solids Struct 2018;150:22-39. https://doi.org/10.1016/j.ijsolstr.2018.05.020.

[3] Khulief Y. Modeling of impact in multibody systems: an overview. J Comput Nonlinear Dyn 2013;8(2):021012https://doi.org/10.1115/1.4006202.
[4] Ugrimov SV, Shupikov AN, Lytvynov LA, Yareshchenko VG. Non-stationary response of sapphire rod on longitudinal impact. Theory and experiment. Int J Impact Eng 2017;104:55-63. https://doi.org/10.1016/j.ijimpeng.2017.02.005.

[5] Païdoussis MP, Li GX. Cross-flow-induced chaotic vibrations of heat-exchanger tubes impacting on loose supports. J Sound Vib 1992;152(2):305-26. https://doi. org/10.1016/0022-460X(92)90363-3.

[6] Belal A, Dongchan J, Sanghoon L. Structural integrity of a high-burnup spent fuel rod under drop impact considering pellet-clad interfacial bonding influence. Nucl Eng Des 2018;337:324-40. https://doi.org/10.1016/j.nucengdes.2018.07.024.

[7] Anderson CE, Orphal DL, Behner T, Templeton DW. Failure and penetration 
response of borosilicate glass during short-rod impact. Int $\mathrm{J}$ Impact Eng 2009;36(6):789-98. https://doi.org/10.1016/j.ijimpeng.2008.12.002.

[8] Anderson CE, Chocron S, Bigger RP. Time-resolved penetration into glass: Experiments and computations. Int J Impact Eng 2011;38(8-9):723-31. https://doi. org/10.1016/j.ijimpeng.2011.03.007.

[9] Ghaednia H, Marghitu DB, Jackson RL. Predicting the permanent deformation after the impact of a rod with a flat surface. J Tribol 2014;137(1):011403https://doi.org/ 10.1115/1.4028709.

[10] Gheadnia H, Cermik O, Marghitu DB. Experimental and theoretical analysis of the elasto-plastic oblique impact of a rod with a flat. Int J Impact Eng 2015;86:307-17. https://doi.org/10.1016/j.ijimpeng.2015.08.007.

[11] Behner T, Anderson Jr. CE, Orphal D, Hohler V, Moll M, Templeton D. Penetration and failure of lead and borosilicate glass against rod impact. Int J Impact Eng 2008;35(6):447-56. https://doi.org/10.1016/j.ijimpeng.2007.04.004.

[12] Ghaednia H, Cermik O, Marghitu DB, Kardel K. Collision measurements using digital image correlation techniques. Int J Mech Sci 2017;131:836-46. https://doi. org/10.1016/j.ijmecsci.2017.07.025.

[13] Li JC, Chen XW, Huang FL. FEM analysis on the "self-sharpening" behavior of tungsten fiber/metallic glass matrix composite long rod. Int J Impact Eng 2015;86:67-83. https://doi.org/10.1016/j.ijimpeng.2015.07.006.

[14] Liu J, Hou B, Lu F, Zhao H. A theoretical study of shock front propagation in the density graded cellular rods. Int J Impact Eng 2015;80:133-42. https://doi.org/10. 1016/j.ijimpeng.2015.02.001.

[15] Marghitu DB, Hurmuzlu Y. Nonlinear dynamics of an elastic rod with frictional impact. Nonlinear Dyn 1996;10(2):187-201. https://doi.org/10.1007/ BF00045457.

[16] Yatteau JD, Dzwilewski PT. Adaptation of full impact penetration models to partial impact geometries for tumbling rods penetrating spaced plates. Int $\mathrm{J}$ Impact Eng 2003;29(1-10):821-31. https://doi.org/10.1016/j.ijimpeng.2003.10.027.

[17] Hertz H. Über Die Berührung Fester Elastischer Körper (on the contact of elastic solids). J Für Die Reine Und Angewandte Mathematik 1882;1882(92):156-71. https://doi.org/10.1515/crll.1882.92.156.

[18] Kogut L, Komvopoulos K. Analysis of the spherical indentation cycle for elasticperfectly plastic solids. J Mater Res 2004;19(12):3641-53. https://doi.org/10. 1557/JMR.2004.0468.

[19] Ye N, Komvopoulos K. Indentation analysis of elastic-plastic homogeneous and layered media: criteria for determining the real material hardness. J Tribol 2003;125(4):685-91. https://doi.org/10.1115/1.1572515.

[20] Brake M. An analytical elastic-perfectly plastic contact model. Int J Solids Struct 2012;49(22):3129-41. https://doi.org/10.1016/j.ijsolstr.2012.06.013.
[21] Stronge WJ. Contact problems for elastoplastic impact in multi-body systems. Impacts in Mechanical Systems. Berlin Heidelberg: Springer; 2000.

[22] Thornton C. Coefficient of restitution for collinear collisions of elastic-perfectly plastic spheres. J Appl Mech 1997;64(2):383-6. https://doi.org/10.1115/1. 2787319.

[23] Jackson RL, Green I. A finite element study of elasto-plastic hemispherical contact against a rigid flat. J Tribol 2005;127(2):343-54. https://doi.org/10.1115/1. 1866166.

[24] Kogut L, Etsion I. Elastic-plastic contact analysis of a sphere and a rigid flat. J Appl Mech 2002;69(5):657-62. https://doi.org/10.1115/1.1490373.

[25] Brake MRW. An analytical elastic plastic contact model with strain hardening and frictional effects for normal and oblique impacts. Int J Solids Struct 2015;62:104-23. https://doi.org/10.1016/j.ijsolstr.2015.02.018.

[26] Ghaednia H, Brake MR, Berryhill M, Jackson RL. Strain hardening from elastic-perfectly plastic to perfectly elastic flattening single asperity contact. J Tribol 2019;141(3):031402https://doi.org/10.1115/1.4041537.

[27] Paoli L, Schatzman M. Numerical simulation of the dynamics of an impacting bar. Comput Meth Appl Mech Eng 2007;196(29-30):2839-51. https://doi.org/10.1016/ j.cma.2006.11.024.

[28] Stoianovici D, Hurmuzlu Y. A critical study of the applicability of rigid-body collision theory. J Appl Mech 1996;63(2):307-16. https://doi.org/10.1115/1. 2788865.

[29] Hurmuzlu Y. An energy-based coefficient of restitution for planar impacts of slende bars with massive external surfaces. J Appl Mech 1988;65(4):952-62. https://doi. org/10.1115/1.2791939.

[30] Kim Y, Kim H, Bak J, Jeong J, Jeon J, Yun B. Evaluation of thermal hydraulic safety of a nuclear fuel assembly in a mast assembly of nuclear power plant. Ann Nucl Energy 2016;92:136-49. https://doi.org/10.1016/j.anucene.2016.01.033.

[31] Gajapathy R, Velusamy K, Selvaraj P, Chellapandi P, Chetal S. CFD investigation of helical wire-wrapped 7-pin fuel bundle and the challenges in modeling full scale 217 pin bundle. Nucl Eng Des 2007;237(24):2332-42. https://doi.org/10.1016/j. nucengdes.2007.05.003.

[32] Lee A, Komvopoulos K. Dynamic spherical indentation of elastic-plastic solids. Int J Solids Struct 2018;146:180-91. https://doi.org/10.1016/j.ijsolstr.2018.03.028.

[33] Lee A, Komvopoulos K. Dynamic spherical indentation of strain hardening materials with and without strain rate dependent deformation behavior. Mech Mater 2019;133:128-37. https://doi.org/10.1016/j.mechmat.2018.12.002.

[34] Kumar B, Sujith R. Exact solutions for the longitudinal vibration of non-uniform rods. J Sound Vib 1997;207(5):721-9. https://doi.org/10.1006/jsvi.1997.1146.

[35] Thomson W. Theory of vibration with applications. CrC Press; 2018. 